\title{
The Lax-Wendroff Theorem of Entropy Dissipation Method for Scalar Conservation Laws in One Space Dimension
}

\author{
Hongxia Li \\ College of Mathematics and Statistics \\ Zhejiang University of Finance and Economics \\ Hangzhou 310018, China \\ E-mail: hxli@zufe.edu.cn
}

Research was supported by China NSF Grant, No. 10871168; Supported by Scientific Research Fund of Zhejiang Provincial Education Department, No.20070825.

\begin{abstract}
In this paper, we present the Lax-Wendroff theorem of entropy dissipation method for scalar conservation laws in one space dimension. Suppose that $u_{l}(x, t)$ the numerical solution computed by the entropy dissipation method converges to a function $u(x, t)$ as $l \rightarrow \infty$,then $\mathrm{u}(x, t)$ is a weak solution that satisfying the entropy condition of the conservation law.
\end{abstract}

Keywords: Conservation law, Entropy condition, Lax-Wendroff theorem

\section{Introduction}

In this paper we continue to consider entropy dissipating method developed in(Li, Hong-xia, 2004), (Secondorder entropy dissipation scheme for scalar conservation laws in one space dimension, Master's thesis, No.1190399118086)for scalar conservation laws in one space dimension

$$
u_{t}+f(u)_{x}=0 \quad u(x, 0)=u_{0}(x)
$$

In this paper, we propose and prove a Lax-Wendroff theorem of entropy dissipation method for scalar conservation laws in one space dimension.

\section{The Basic Definitions}

In this section, we give the basic definitions of the theorem. We will consider the general form of the scheme.The numerical solution is computed by:

$$
u_{j}^{n+1}=u_{j}^{n}-\lambda\left(\hat{f}_{j+\frac{1}{2}}^{n}-\hat{f}_{j-\frac{1}{2}}^{n}\right)
$$

where the numerical flux is:

$$
\hat{f}_{j+\frac{1}{2}}^{n}=\hat{f}\left(u_{j-k+1}^{n}, \cdots, u_{j+k}^{n} ; U_{j-p+1}^{n}, \cdots, U_{j+p}^{n}\right)
$$

The numerical entropy is computed by:

$$
U_{j}^{n+1}=U_{j}^{n}-\lambda\left(\hat{F}_{j+\frac{1}{2}}^{n}-\hat{F}_{j-\frac{1}{2}}^{n}\right)-D_{j}^{n}
$$

where the numerical entropy flux is:

$$
\hat{F}_{j+\frac{1}{2}}^{n}=\hat{F}\left(u_{j-k+1}^{n}, \cdots, u_{j+k}^{n} ; U_{j-p+1}^{n}, \cdots, U_{j+p}^{n}\right)
$$




$$
D_{j}^{n}=D\left(u_{j-l}^{n}, \cdots, u_{j+l}^{n}\right)
$$

$k, p, l$ are the positive integers.

Definition 2.1 (consistence): If all the $u_{i}^{n}(i=\max (j-k+1, j-l), \cdots, \max (j+k, j+l))$ in (3), (5), (6) are $\bar{u}$ and all the $U_{i^{\prime}}^{n}\left(i^{\prime}=j-p+1, \cdots, j+p\right)$ are $U(\bar{u})$, (where $\bar{u} \in R$ ), then:

$$
\begin{gathered}
\hat{f}(\bar{u}, \cdots, \bar{u} ; U(\bar{u}), \cdots, U(\bar{u}))=f(\bar{u}) \\
\hat{F}(\bar{u}, \cdots, \bar{u} ; U(\bar{u}), \cdots, U(\bar{u}))=F(\bar{u}) \\
D(\bar{u}, \cdots, \bar{u})=0
\end{gathered}
$$

If $u_{i} \rightarrow \bar{u}, U_{i^{\prime}} \rightarrow U(\bar{u})$, then $\hat{f}, \hat{F}$ and $D$ convergence to $f(\bar{u}), F(\bar{u})$ and 0 in the following: for $0<q \leq 1$ there is a constant $K$ (maybe dependent $\bar{u}$ ) such that at $\bar{u}$ :

$$
\begin{gathered}
\left|\hat{f}\left(u_{j-k+1}, \cdots, u_{j+k} ; U_{j-p+1}, \cdots, U_{j+p}\right)-f(\bar{u})\right| \\
\leq K \max _{\substack{j-k+1 \leq i \leq j+k \\
j-p+1 \leq i^{\prime} \leq j+p}}\left(\left|u_{i}-\bar{u}\right|,\left|U_{i^{\prime}}-U(\bar{u})\right|^{q}\right) \\
\left|\hat{F}\left(u_{j-k+1}, \cdots, u_{j+k} ; U_{j-p+1}, \cdots, U_{j+p}\right)-F(\bar{u})\right| \\
\quad \leq K \max _{\substack{j-k+1 \leq i \leq j+k \\
j-p+1 \leq i^{\prime} \leq j+p}}\left(\left|u_{i}-\bar{u}\right|,\left|U_{i^{\prime}}-U(\bar{u})\right|^{q}\right) \\
\left|D\left(u_{j-l}, \cdots, u_{j+l}\right)-D(\bar{u}, \cdots, \bar{u})\right| \leq K \max _{j-l \leq i^{\prime \prime} \leq j+l}\left(\left|u_{i^{\prime \prime}}-\bar{u}\right|\right)
\end{gathered}
$$

then the scheme is consistent.

We are going to discuss the theorem as the form in (LeVeque, R.J. , 2002), (LeVeque, R.J., 1990). First we define two piecewise constant function $u_{l}(x, t), U_{l}(x, t)$ for all $x$ and $t$ from the discrete values $\left\{u_{j}^{n}\right\}$ and $\left\{U_{j}^{n}\right\}$ :

$$
u_{l}(x, t)=u_{j}^{n}, U_{l}(x, t)=U_{j}^{n}, \quad x_{j-\frac{1}{2}}<x \leq x_{j+\frac{1}{2}} \quad, \quad t_{n}<t \leq t_{n+1}
$$

\section{The New Lax-Wendroff Theorem}

Theorem 3.1 (Lax-Wendroff): Consider a sequence of $\mathrm{g}$ rids indexed by $l=1,2, \ldots \cdots$, with mesh parameters $k_{l}, h_{l} \rightarrow 0$ as $l \rightarrow \infty$. Let $u_{l}(x, t), U_{l}(x, t)$ are the numerical approximation computed with the scheme (2) (5). Suppose that $u_{l}(x, 0), u_{l}(x, t), U_{l}(x, t)$ are uniformly bounded functions and converge to the functions $u(x, 0)$, $u(x, t), U(u(x, t))$ as $l \rightarrow \infty$, in the sense made precise below. Then $u(x, t)$ is a entropy satisfying weak solution of the conservation law.

As in (R.J. LeVeque, 2002), (R.J. LeVeque, 1990), we assume that we have convergence of $u_{l}(x, t), U_{l}(x, t)$ to $u(x, t), U(u(x, t))$ in the following sense:

on $\Omega=[a, b] \times[0, t](a \leq b, t \geq 0)$, as $l \rightarrow \infty$ :

$$
\begin{gathered}
\int_{0}^{t} \int_{a}^{b}\left|u_{l}(x, t)-u(x, t)\right| d x d t \rightarrow 0 \\
\int_{0}^{t} \int_{a}^{b}\left|U_{l}(x, t)-U(u(x, t))\right| d x d t \rightarrow 0
\end{gathered}
$$

As $l \rightarrow \infty$ :

$$
\begin{gathered}
\left\|u_{l}-u\right\|_{1, \Omega} \rightarrow 0 \\
\left\|U_{l}-U\right\|_{1, \Omega} \rightarrow 0
\end{gathered}
$$

Proof: We will show that the limit function $u(x, t)$ satisfies the weak form, for all $\phi \in C_{0}^{1}\left(R^{2}\right), u(x, t)$ :

$$
\int_{0}^{\infty} \int_{-\infty}^{+\infty}\left(\phi_{t} u+\phi_{x} f(u)\right) d x d t=-\int_{-\infty}^{\infty} \phi(x, 0) u(x, 0) d x
$$


Let $\phi$ be a $C_{0}^{1}\left(R^{2}\right)$ test function and multiply the numerical method (2) by $\phi\left(x_{j}, t_{n}\right)$ and sum it over all $j$ and $n \geq 0$. We obtain

$$
\sum_{n=0}^{\infty} \sum_{j=-\infty}^{\infty} \phi\left(x_{j}, t_{n}\right)\left(u_{j}^{n+1}-u_{j}^{n}\right)=-\frac{k}{h} \sum_{n=0}^{\infty} \sum_{j=-\infty}^{\infty} \phi\left(x_{j}, t_{n}\right)\left(\hat{f}_{j+\frac{1}{2}}^{n}-\hat{f}_{j-\frac{1}{2}}^{n}\right)
$$

we now use "summation by parts", and multiply it by $h$ :

$$
\begin{gathered}
h k\left\{\sum_{n=1}^{\infty} \sum_{j=-\infty}^{\infty} \frac{\phi\left(x_{j}, t_{n}\right)-\phi\left(x_{j}, t_{n-1}\right)}{k} u_{j}^{n}+\sum_{n=1}^{\infty} \sum_{j=-\infty}^{\infty} \frac{\phi\left(x_{j+1}, t_{n}\right)-\phi\left(x_{j}, t_{n}\right)}{h} \hat{f}_{j+\frac{1}{2}}^{n}\right\} \\
=-h \sum_{j=-\infty}^{\infty} \phi\left(x_{j}, 0\right) u_{j}^{0}
\end{gathered}
$$

By our assumption that $\phi$ has compact support, and hence each of the sums is in fact a finite sum.

Since $u_{l}(x, 0), u_{l}(x, t)$ are converge to $u(x, 0), u(x, t)$ in $L^{1}$, and $\phi(x, t)$ is smooth, we get the first term of (20) is converges to $\int_{0}^{\infty} \int_{-\infty}^{+\infty} \phi_{t}(x, t) u(x, t) d x d t$ and the third term converges to $-\int_{-\infty}^{\infty} \phi(x, 0) u(x, 0) d x$. The second term can be written as:

$$
\begin{gathered}
h k \sum_{n=1}^{\infty} \sum_{j=-\infty}^{\infty} \frac{\phi\left(x_{j+1}, t_{n}\right)-\phi\left(x_{j}, t_{n}\right)}{h} \hat{f}_{j+\frac{1}{2}}^{n}=h k \sum_{n=1}^{\infty} \sum_{j=-\infty}^{\infty} \frac{\phi\left(x_{j+1}, t_{n}\right)-\phi\left(x_{j}, t_{n}\right)}{h} f\left(u_{j}^{n}\right) \\
+h k \sum_{n=1}^{\infty} \sum_{j=-\infty}^{\infty} \frac{\phi\left(x_{j+1}, t_{n}\right)-\phi\left(x_{j}, t_{n}\right)}{h}\left(\hat{f}_{j+\frac{1}{2}}^{n}-f\left(u_{j}^{n}\right)\right)
\end{gathered}
$$

Since $f$ is continuous and the above conditions, the first term of (21) converges to $\int_{0}^{\infty} \int_{-\infty}^{\infty} \phi_{x} f(u(x, t)) d x d t$ as $l \rightarrow \infty$. Next we will prove that the second term of the right (3.8) converges to 0 . Because of $\hat{f}$ 's consistence, and $\phi$ has compact support. $\phi$ is continuous different, e.t. there is a $N>0$, such that $\left|\frac{\partial \phi(x, t)}{\partial x}\right| \leq N,(x, t) \in R^{2}$. So:

$$
\begin{aligned}
& \left|h k \sum_{n=1}^{\infty} \sum_{j=-\infty}^{\infty} \frac{\phi\left(x_{j+1}, t_{n}\right)-\phi\left(x_{j}, t_{n}\right)}{h}\left(\hat{f}_{j+\frac{1}{2}}^{n}-f\left(u_{j}^{n}\right)\right)\right| \\
& \quad \leq K N\left\{\sum_{i=j-k+1}^{j+k}\left(h k \sum_{n=1}^{\infty} \sum_{j=-\infty}^{\infty}\left|u_{i}^{n}-u_{j}^{n}\right|\right)+\sum_{i^{\prime}=j-p+1}^{j+p}\left(h k \sum_{n=1}^{\infty} \sum_{j=-\infty}^{\infty}\left|U_{i^{\prime}}^{n}-U\left(u_{j}^{n}\right)\right|^{q}\right)\right\}
\end{aligned}
$$

Due to (13), (14), the right of the above formulas is:

$$
\begin{aligned}
& =K N\left\{\sum_{i=j-k+1}^{j+k}\left(\int_{0}^{t} \int_{a}^{b}\left|u_{l}(x, t)-u_{l}(x+(i-j) h, t)\right| d x d t\right)\right. \\
& \left.+\sum_{i^{\prime}=j-p+1}^{j+p}\left(\int_{0}^{t} \int_{a}^{b}\left|U_{l}(x, t)-U\left(u_{l}\left(x+\left(i^{\prime}-j\right) h, t\right)\right)\right|^{q} d x d t\right)\right\},
\end{aligned}
$$

it includes: $I_{h_{1}}=\int_{0}^{t} \int_{a}^{b}\left|u_{l}(x, t)-u_{l}\left(x+s_{1} h, t\right)\right| d x d t, I_{h_{2}}=\int_{0}^{t} \int_{a}^{b}\left|U_{l}(x, t)-U\left(u_{l}\left(x+s_{2} h, t\right)\right)\right|^{q} d x d t$. where $-k+1 \leq$ $s_{1} \leq k,-p+1 \leq s_{2} \leq p$ is the positive integer, note:

$$
\begin{aligned}
I_{h_{1}} & \leq \int_{0}^{t} \int_{a}^{b}\left|u_{l}(x, t)-u(x, t)\right| d x d t+\int_{0}^{t} \int_{a}^{b}\left|u(x, t)-u\left(x+s_{1} h, t\right)\right| d x d t \\
& +\int_{0}^{t} \int_{a}^{b}\left|u\left(x+s_{1} h, t\right)-u_{l}\left(x+s_{1} h, t\right)\right| d x d t,
\end{aligned}
$$

Since $u_{l}(x, t)$ converging to $u(x, t)$ in $L^{1}$, as $l \rightarrow \infty$, the right term of the above formulas $\rightarrow 0$. Using $|a+b+c|^{q} \leq 3^{q}\left(|a|^{q}+|b|^{q}+|c|^{q}\right), q \geq 0$ we get:

$$
\begin{aligned}
I_{h_{2}} & \leq 3^{q}\left\{\int_{0}^{t} \int_{a}^{b}\left|U_{l}(x, t)-U(u(x, t))\right|^{q} d x d t+\int_{0}^{t} \int_{a}^{b}\left|U(u(x, t))-U\left(u\left(x+s_{2} h, t\right)\right)\right|^{q} d x d t\right. \\
& \left.+\int_{0}^{t} \int_{a}^{b}\left|U\left(u\left(x+s_{2} h, t\right)\right)-U\left(u_{l}\left(x+s_{2} h, t\right)\right)\right|^{q} d x d t\right\} .
\end{aligned}
$$


Due to Hölder inequality, $\int_{0}^{t} \int_{a}^{b}|f(x, t)|^{q} d x d t$ can be controlled by $\int_{0}^{t} \int_{a}^{b}|f(x, t)| d x d t$. Since $U_{l}(x, t) \rightarrow U(u(x, t))$ in $L^{1}$, right of the above inequality $\rightarrow 0$, as $l \rightarrow \infty$.

Above all the second term of the right of $(21) \rightarrow 0$, as $l \rightarrow \infty$. So $u(x, t)$ satisfies:

$$
\int_{0}^{\infty} \int_{-\infty}^{\infty}\left(\phi_{t} u(x, t)+\phi_{x} f(u(x, t))\right) d x d t=-\int_{-\infty}^{\infty} \phi(x, 0) u(x, 0) d x d t .
$$

The limit function $u(x, t)$ is a weak solution of the conservation law.

We can prove the solution also satisfies the entropy condition in the same way. Note $D_{j}^{n} \geq 0$ and $\phi(x, t)>0 \in$ $C_{0}^{1}\left(R_{+}^{2}\right)$.

\section{References}

R.J. LeVeque, Finite volume methods for hyperbolic problems, Published by the press Syndicate of the University of Cambridge, (2002).

R.J. LeVeque, Numerical methods for conservation laws, Birkhauser-verla, Basel, Boston, Berlin, (1990).

Li, Hong-xia; Mao De-kang,Further development of a entropy dissipating method for scalar conservation laws. J. Inform. Comput. Sci. 147, 1(3), (2004).

Second-order entropy dissipation scheme for scalar conservation laws in one space dimension, Master's thesis, No. 11903-99118086, Shanghai University (in Chinese). 